\title{
On the Partial Filling of the Intermediate Band in IB Solar Cells
}

\author{
Antonio Luque, Senior Member, IEEE, and Antonio Martí
}

\begin{abstract}
Based on a generalized model of the ShockleyRead-Hall (SRH) statistics published elsewhere, the effect of the partial filling of the intermediate band (IB) in IB solar cells and the ways of producing it are analyzed, as is its influence on the electron-hole pair generation by subband-gap photons. The differences between cells with the conduction band and the IB thermally coupled and uncoupled are stressed. This paper is oriented toward the explanation of the operation of quantum-dot solar cells, where the IB is formed from electron-confined states but can also be applicable to other IB systems.
\end{abstract}

Index Terms-Intermediate band (IB), quantum dot (QD), solar cell.

\section{INTRODUCTION}

$\mathbf{T}$ HE OBJECTIVE of the intermediate-band (IB) solar cell $[1],[2]$ is to increase the photocurtent of a solar cell while keeping the voltage almost unchanged. This is produced by the successive (and independent) pumping of an electron from the valenee band (VB) to the IB with a subband-gap energy photon and another electron from the IB to the conduction band (CB) with another subband-gap energy photon (Fig. 1). Thus, the net process is the generation of an electron-hole pair from two subband-gap energy photons. As in conventional cells, the voltage is still the difference (in electronvolts) of quasiFermi levels of the CB electrons at the $n$ contact and the VB electrons at the p contact. The IB has its own quasi-Fermi level $F_{t}$. For this cell to appropriatcly work, it is necessary for the IB to be hal[-filled [1], [2] to find emply states to accommodate the electrons proceeding from the $\mathrm{CB}$ and filled states to permit electrons to $\mathrm{be}$ pumped to the $\mathrm{CB}$. In quantumdot (QD) solar cells, the half-filling of the IB is usually achieved by doping the IB region. However, in many cases, researchers are reluctant to carry out this doping possibly because they consider it will degrade the cell. Furthermore, this strategy is often confirmed, in their view, by the achievement of better quantum efficiency. We shall show that, in general, not having the IB partially filled increases the cell photocurrent when the IB is connected to the CB (in the sense of the IB exhibiting

Manuscript received August 7. 2009; revised March 1, 2010; accepted March 3, 2010. Dale of publicalion April 15, 2010; date of current version May 19,2010 This work was supported in part by the European Commission through Project IBPOWER under Contract 211640 , by the Conunidad de Madrid through Project NUMANCIA under Contract S2009/ENE/1477. and by the Spanish National Program through Project GENESIS-FV under Contract CSD2006-00004. The review of this paper was arranged by Editor P. Panayotatos.

The authors are with the Instituto de Energía Solar, Universidad Politécnica de Madrid, ETSI Telecomunicación, 28040 Madrid, Spain (e-mail: amarti@etsit.upm.es).

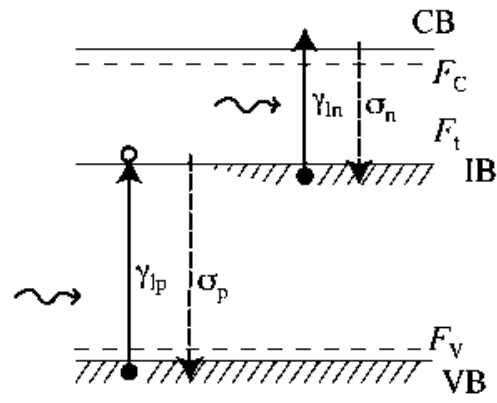

Fig. 1. Simplified energy band-gap diagram of an IB material showing the photon absorption and electron and hole capture processes involving the IB.

a high-capture cross section for electrons in the $\mathrm{CB}$ ), which can misleadingly lead to the conclusion of having achieved a cell with increased efficiency potential. We will show that this is not the case since, having the IB and the CB connected, a voltage drop will accompany the increase in photocurrent in good cells and an increase in efficiency will not be produced. On the contrary, if the IB and the CB are not connected, although the cell will exhibit a lower increase in photocurrent than that of the connected cell (which can be solved by making the IB thicker), this increase will not be followed, in particular under concentrated light, by an excessive drop in voltage, which results in a significant potential increase in efficiency.

The increase of the photocurrent based on light absorption by impurities has been proposed by several authors since 1960 [3], [4]. Impurity photovoltaics, with impurity levels rather than an IB, has been studied more recently at the light of the IB solar ecll [5], [6]. The use of the term IB comes from the convenience of increasing the density of impurities above the Mott transition to prevent [7] the nonradiative recombination based in the lattice-relaxation multiphonon emission mechanism [8]. The reason is that, in this mechanism, recombination through localized states involves a local change in the electric charge density that is responsible of the displacement of the impurity from its equilibrium position and therefore favors the recombination by means of the emission of multiple phonons. However, in this paper, no conduction is considered along the IB. In fact, we are thinking of the energy levels introduced by the QDs as confined levels. In QDs, since the confined electrons are already extended, the formation of bands is unnecessary to reduce nonradiative recombination. The results in this paper are equally valid for cells with bands or levels due to impurities.

\section{IB FILLING FACTOR}

What we call an IB may in fact be a complex structure. If it is formed from QD confined states, several energy levels 
TABLE I

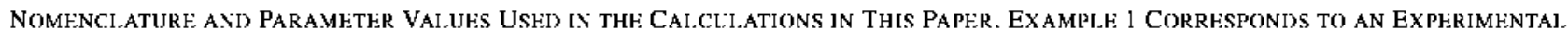

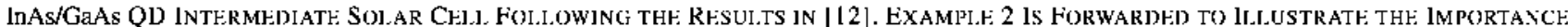

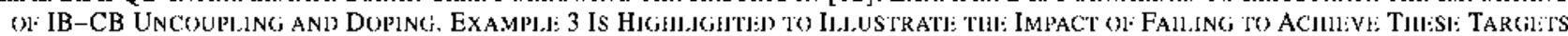

\begin{tabular}{|c|c|c|c|c|}
\hline Symbol & Description & & Cell examples & \\
\hline & & $\begin{array}{l}\text { One sun narrow coupled } \\
\text { IB-CB (Example 1) }\end{array}$ & $\begin{array}{l}1000 \text { suns uncoupled } \\
\text { IB-CB (Example 2) }\end{array}$ & $\begin{array}{l}1000 \text { suns wide coupled } \\
\text { IB-CB (Example } 3 \text { ) }\end{array}$ \\
\hline$\sigma_{n}\left(\mathrm{~cm}^{2}\right)$ & Electron capture cross section & $2.35 \times 10^{-12}$ & $7.83 \times 10^{-18}$ & $7.83 \times 10^{-13}$ \\
\hline$\sigma_{p}\left(\mathrm{~cm}^{2}\right)$ & Hole capture cross section & $3 \times 10^{-14}$ & $3 \times 10^{-17}$ & $3 \times 10^{-17}$ \\
\hline$N_{C}\left(\mathrm{~cm}^{-3}\right)$ & Effective density of states at the CB & $4.7 \times 10^{17}$ & $4.7 \times 10^{17}$ & $4.7 \times 10^{17}$ \\
\hline$N_{V}\left(\mathrm{~cm}^{-3}\right)$ & Effective density of states at the VB & $7 \times 10^{18}$ & $7 \times 10^{18}$ & $7 \times 10^{18}$ \\
\hline$N_{t}\left(\mathrm{~cm}^{-3}\right)$ & Densily of Iraps & $8 \times 10^{16}$ & $1 \times 10^{18}$ & $1 \times 10^{18}$ \\
\hline$N_{D}\left(\mathrm{~cm}^{-3}\right)$ & Donor density & $4 \times 10^{16}$ & $5 \times 10^{17}$ & 0 \\
\hline$E_{g}(\mathrm{eV})$ & Bandgap & 1.41 & 1.41 & 1.41 \\
\hline$E_{l}(\mathrm{eV})$ & IB position (measured from the VB) & 1.13 & 1.13 & 1.13 \\
\hline$\phi_{B}(\mathrm{~V})$ & Potential barricr & 1.3613 & 1.3613 & 1.3613 \\
\hline$\gamma_{l n}\left(\mathrm{~cm}^{-1}\right)$ & Electron generation coefficient & $2.31 \times 10^{-4}$ & $7.83 \times 10^{-2}$ & $7.83 \times 10^{-2}$ \\
\hline$\gamma_{l p}\left(\mathrm{~cm}^{-1}\right)$ & Hole generation coefficient & $2.31 \times 10^{-4}$ & $1.18 \times 10^{-1}$ & $1.18 \times 10^{-1}$ \\
\hline$W(\mathrm{~nm})$ & Thickness & 100 & 1185 & 592 \\
\hline
\end{tabular}

may appear depending on the shape and size of the QD. Up to four confined levels followed by higher levels that merge into the $\mathrm{CB}$ can be distinguished below the $\mathrm{CB}$ in GaAs with InAs QDs either experimentally [9] or by modeling [10], [11] (although the result is dependent on the size and shape of the QD). Only the fundamental confined electron state is usually designated as an IB. Above these levels, more closely spaced confined-state levels merge into the $\mathrm{CB}$. As regards the holes, confined states are also present, but due to their usually higher effective mass, they are more closely spaced and merge into the VB. Therefore, the optical band gap may be reduced by levels merging with the VB and the $\mathrm{CB}$ as described. Using this reduction blindly may lead to an error because the density of states of these levels is much smaller than the (equivalent) one in the CB (on the order of $10^{17}-10^{19} \mathrm{~cm}^{-3}$ ). For the purpose of calculating the clectron and hole density, we prefer to leave the band edges unchanged and to account for the merging QD states as a factor that increases the carrier capture sections of electrons and holes. Because of this complexity, a simplified model is a good guide for the development of effective IB solar cells [12]-[14].

In our model, the IB solar cell is made up of a p-type semiconductor (that we will locate on the left of our plots); an IB region with VB, $C B$, and a single IB; and an n-type semiconductor (on the right). The IB region may be doped (with donors in our case) or not doped. This simplified model is a generalized Shockley-Read-Hall (GSRH) model that has been developed in [12]. Essentially, it is assumed that the IB is formed by deep levels brought about by impurities or QDs that can interchange electrons with the $\mathrm{CB}$ and the VB through the processes of electron and hole capture and emission mechanisms. In addition, in this GSRH model, the deep levels absorb photons from a source out of the equilibrium (e.g., the sun) through the emission of holes to the VB (pumping electrons from the VB) or the emission of electrons to the CB. The photons in equilibrium are assumed to be included in the ordinary emission and capture mechanisms. Taking all this into account, following a scheme parallel to that followed by Shockley and Read [15] and Hall [16] and improved by Keevers and Green [4] and Hsieh and Hard [17],
Luque et al. [12] obtain an expression for the filling factor $f$ of the IB, i.e.,

$$
f=\frac{f_{n}}{f_{d}}
$$

where

$$
\begin{aligned}
f_{n}=\sigma_{\pi b} N_{C} \exp \left(-\frac{E_{g}-e \varphi-\Delta_{\mathrm{FV}}-e V}{k T}\right) \\
+\sigma_{p} N_{V} \exp \left(-\frac{E_{t}}{k T}\right)+\gamma_{\mathrm{p}} \\
f_{d}=\sigma_{n t} N_{C}\left[\exp \left(-\frac{E_{g}-e \varphi-\Delta_{\mathrm{FV}}-e V}{k T}\right)\right. \\
\left.+\exp \left(-\frac{E_{g}-E_{t}}{k T}\right)\right] \\
+\sigma_{p} N_{V}\left[\exp \left(-\frac{\Delta_{\mathrm{FV}}+e \varphi}{k T}\right)\right. \\
\left.+\exp \left(-\frac{E_{t}}{k T}\right)\right]+\gamma_{\mathrm{ln}}+\gamma_{\mathrm{lp}} .
\end{aligned}
$$

The notation is defined in Table I, where the cases being studied that will appear in this paper are also listed. These cases essentially correspond to an IB cell operated at one sun (Example 1) and at 1000 suns. In addition, the case of operation at 1000 suns is split in two, known as IB-CB coupled (Example 2) and uncoupled (Example 3). IB-CB coupled is defined by a high value of the clectron capture cross section $\sigma_{n}$, whereas the IB-CB uncoupled case corresponds to a low value of this parameter. Temperature $T$ will be $300 \mathrm{~K}$ in all cases.

The origin of the $x$-coordinate and of the potential $\varphi$ are taken at the interface between the $\mathrm{p}$ region (on the left in our drawings) and the IB region. The origin of the energies is taken at the top of the VB at $x=0$. The electron and hole generation coefficients $\gamma_{\mathrm{ln}}$ and $\gamma_{\mathrm{p}}$ are given, respectively, by

$$
\begin{aligned}
& v_{\mathrm{th}} \gamma_{\mathrm{ln}} N_{t}=\int \alpha_{\mathrm{IC}}^{(0)}\left(E_{\mathrm{ph}}\right) F_{\mathrm{ph}}\left(E_{\mathrm{ph}}\right) d E_{\mathrm{pht}} \\
& v_{\mathrm{th}} \gamma_{\mathrm{lp}} N_{t}=\int \alpha_{\mathrm{VI}}^{(0)}\left(E_{\mathrm{ph}}\right) F_{\mathrm{ph}}\left(E_{\mathrm{ph}}\right) d E_{\mathrm{ph}}
\end{aligned}
$$


with $\alpha_{\mathrm{IC}}^{(0)}=\alpha_{1 \mathrm{C}}\left(E_{\mathrm{ph}}\right) / f$, and $\alpha_{\mathrm{VI}}^{(0)}=\alpha_{\mathrm{VI}}\left(E_{\mathrm{ph}}\right) /(1-f)$. Notice that the absorption coefficients $\alpha_{X Y}$, where $X Y$ represents the absorption from the $X$ band to the $Y$ band, depend on the trap-filling factor (1). The terms with the index zero represent the most favorable condition for the absorption coefficient, which, for VB $\rightarrow$ IB transitions, is that in which all the traps are emply and, for IB $\rightarrow$ CB transitions, is that in which all the traps are filled.

Equation (1) has a dependenec with $x$ through $\varphi . \Delta_{F V}$ is the VB quasi-Fermi level measured from the top of the VB at $x=0$. It might also be position dependent (through $\varphi$ ). At $x=0$, it takes a small value associated with the doping of the $\mathrm{p}$ region. The CB quasi-Fermi level is represented by $\Delta_{\mathrm{FV}}+V$, where $V$ is the local quasi-Fermi level split. Consequently, $V$ may also be position dependent and, therefore, is not generally the applied voltage. $V$ becomes the applied voltage once we ignore the spatial variation of the quasi-Fermi levels, as we do in this paper. Assuming the CB and VB quasi-Fermi levels to be constant also implies assuming a high clectron and hole mobility and, therefore, that ohmic losses are neglected. Notice, however, that the IB quasi-Fermi is not assumed to be constant in this paper but to vary as required to satisfy the proper value of the filling factor. Any degeneracy or high doping effect can be included in the model by considering potential-dependent effective densities of states $N_{C}^{*}(\varphi, x(\varphi))$ and $N_{V}^{*}(\varphi, x(\varphi))$.

The generation coefficients depend on the photon flux and, through it, also on the position, the concentration level, and the spectral photon distribution between the energies involved in the IB $\rightarrow$ CB and VB $\rightarrow$ IB transitions. However, the dependence on the position is negligible if the absorption is weak. Even if it is not, for the sake of simplicity, we have taken the light flux as independent of the depth. With all these simplifications, $f$, which is explicitly dependent on $\varphi$ in (1)-(3), becomes independent of the position, except through $\varphi(x)$.

We plot the filling factor $f(\varphi)$ for several values of $V$ in Fig. 2. The IB is almost empty for low values of the electrostatic potential $\varphi$ and is almost full for the highest values. The threshold, the potential at which a rapid increase in the filling factor takes place, depends on the quasi-Fermi level split $V$.

In (2) and (3), the terms $N_{C} \exp \left(-\left(E_{g}-e \varphi-\Delta_{\mathrm{FV}}-\right.\right.$ $e V / k T))$ and $N_{V} \exp \left(-\left(\Delta_{\mathrm{FV}}+e \varphi / k T\right)\right)$ represent the densities of electrons and holes, respectively. These take high values near the $\mathrm{n}$ region (accumulation of electrons) and the $\mathrm{p}$ region (accumulation of holes), respectively, that sandwich the IB region. The hole density appears in the denominator, and the term containing it is dominant for a small $\varphi$, that is, near the $p$ region, making $f$ close to zero. The electron density appears in the numerator and the denominator, and the terms containing it are dominant for $\varphi$ close to $\Phi_{B}-V$, that is, near the $n$ region, thus making $f$ close to 1 . Far from the edges, both terms strongly decrease to become negligible.

There are also terms that are independent of the potential. These are $\sigma_{p} N_{V} \exp \left(-\left(E_{t} / k T\right)\right)$ and $\sigma_{n} N_{G} \exp \left(-\left(E_{g}-\right.\right.$ $\left.E_{t} / k T\right)$ ) on one side and $\gamma_{\mathrm{p}}$ and $\gamma_{\mathrm{n}}$ on the other. Among these terms, $\sigma_{p} N_{V} \exp \left(-\left(E_{t} / k T\right)\right)$ is totally negligible in the case of cells made of InAs QDs in GaAs (given the large value for $E_{t}$ ), so that in the one-sun cell [Fig. 2(a)], at intermediate values of the potential where the accumulation of holes and electrons has

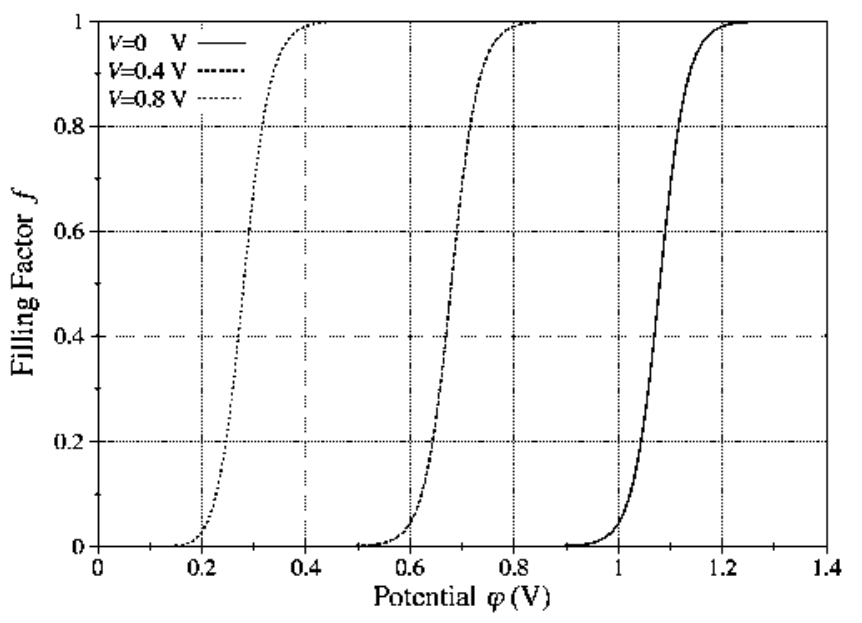

(a)

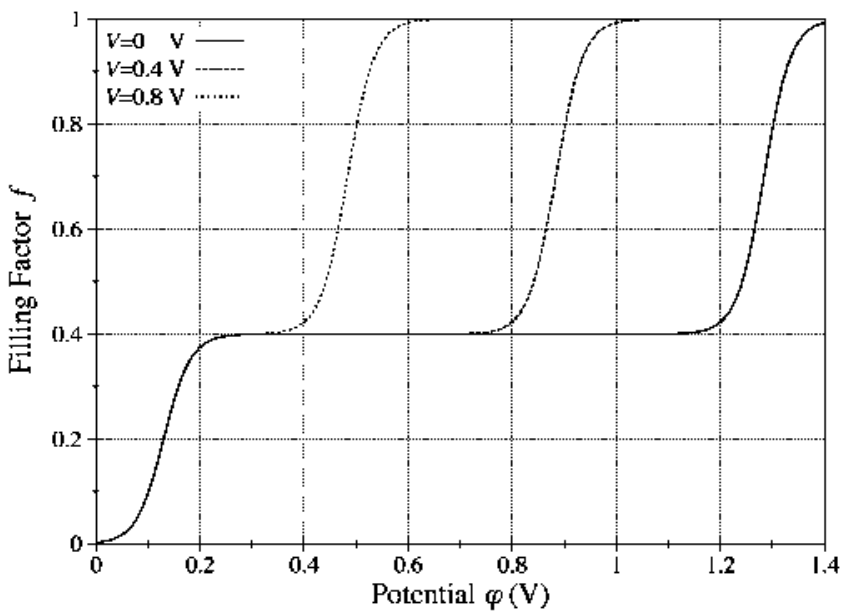

(b)

Fig. 2. Filling factor $f$ versus potential $\varphi$ for several values of the applied voltage $V$. Note that the highest $\varphi$ value for every function is $\Phi_{H}-V$. (a) Example 1: one sun, narrow IB, IB-CB coupled cell (Table I) (b) Example 2: 1000 suns, wide $\mathrm{lB}, \mathrm{lB}-\mathrm{CB}$ uncoupled cell (Table 1).

disappeared, $f \cong \gamma_{\mathrm{p}} / \sigma_{n} N_{C} \exp \left(-\left(E_{g}-E_{t}\right) / k T\right)=2.31 \times$ $10^{-4} / 21.8=1.05 \times 10^{-5}$. On the contrary, for the IB-CB uncoupled concentration case, the term containing $\sigma_{n}$ is reduced because of the smaller $\sigma_{n}$ value and the higher values $\gamma_{\mathrm{p}}$ and $\gamma_{\mathrm{ln}_{\mathrm{n}}}$. Therefore, outside the accumulation regions, $f \cong$ $\gamma_{\mathrm{p}} /\left(\gamma_{l_{\mathrm{n}}}+\gamma_{\mathrm{p}}\right)=0.4$, as found in Fig. 2(b).

The interpretation of these results is that the filling of the IB, for a wide range of potentials, is governed by the balance between the filling of the IB traps and their emplying. In the one-sun IB-CB coupled case, the filling mechanism is the pumping of electrons from the VB to the IB through photon absorption, and the emptying is by thermal escape. In the 1000sun uncoupled $\mathrm{IB}-\mathrm{CB}$ case, the filling is by photon pumping through the $\mathrm{VB} \rightarrow \mathrm{IB}$ transition $\left(\gamma_{\mathrm{p}}(1-f)\right)$ and the emptying by photon pumping through the IB $\rightarrow$ CB transition $\left(\gamma_{1 n} f\right)$.

\section{BAND Diagrams}

However, the fact that, in a large part of the $\varphi$-domain, the filling of the IB is governed by the balance that we have just explained does not imply that this has to be the case, 


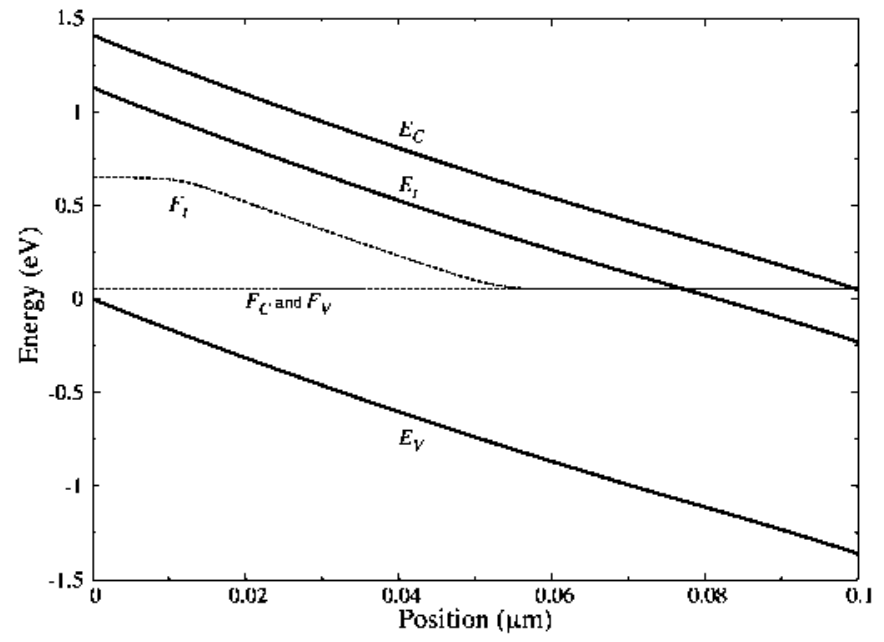

(a)

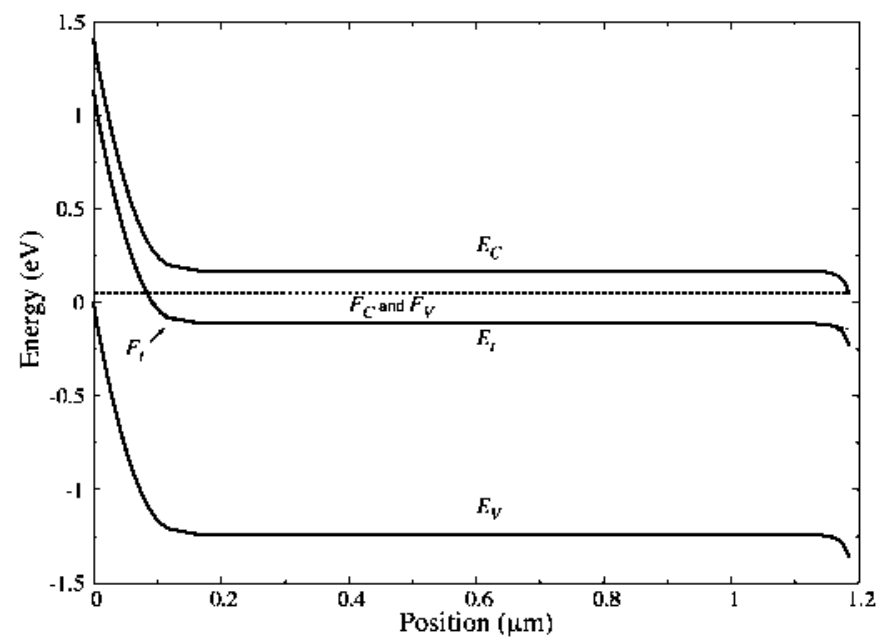

(c)

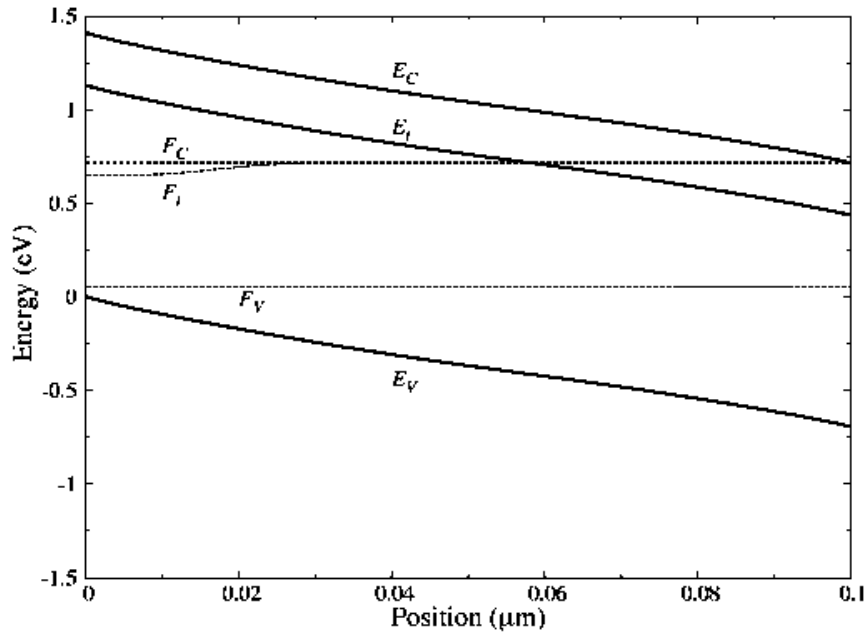

(b)

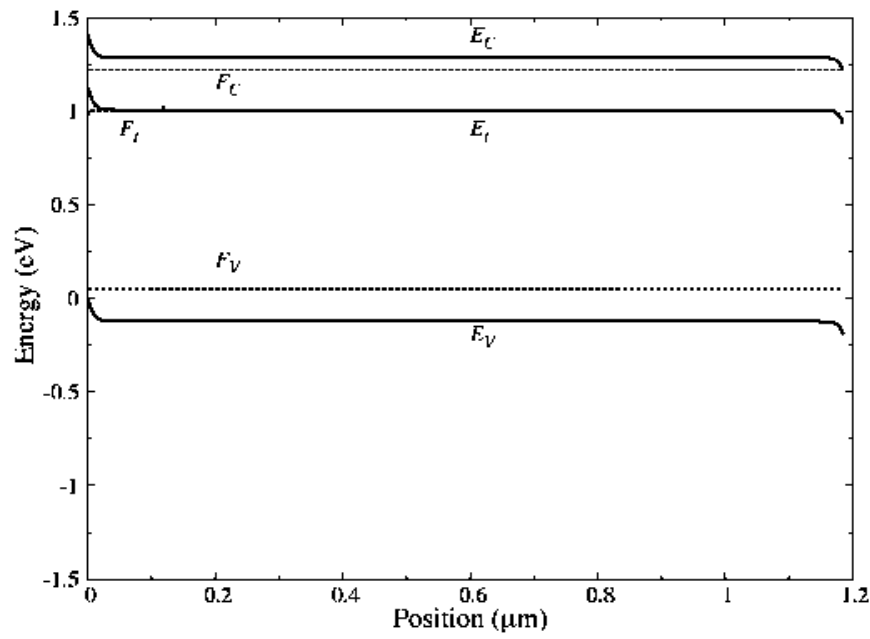

(d)

Fig. 3. Band diagram showing the position of the (solid lines) $\mathrm{CB}, \mathrm{VB}$, and $\mathrm{IB}$ (or $E_{i}$ ) and (dashed lines) quasi-Fermi levels $F_{C}, F_{V}$, and $F_{t}$ corresponding to the electrons in the CV, VB, and IB, respectively. (a) One sun, narrow IB, IB-CB coupled cell (Example 1) at short circuit. (b) At open circuit ( $V=0.667 \mathrm{~V})$. (c) One thousand suns, wide IB, IB-CB uncoupled cell (Example 2) at short circuit. (d) Open circuil $(V=1.17 \mathrm{~V})$.

when translated through the relationship $\varphi(x)$, for most of the $x$-domain. In this respect, let us examine the Poisson equation

$$
\begin{aligned}
& \frac{d \xi}{d x}=\frac{\rho}{\epsilon \epsilon_{0}}=\frac{e}{\epsilon \epsilon_{0}}\left[N_{V} \exp \left(-\frac{\Delta_{\mathrm{FV}}+e \varphi}{k T}\right)\right. \\
& \left.-N_{C} \exp \left(-\frac{E_{g}-e \varphi-\Delta_{\mathrm{FV}}-e V}{k T}\right)+N_{D}-N_{t} f\right]
\end{aligned}
$$

where $\xi$ is the electric field. The space charge is strongly positive in the region close to the p contact (the region with accumulated holes) and strongly negative in the region close to the $\mathrm{n}$ contact (the region with accumulated electrons). At some point in between, it becomes zero. If the IB is thin, this zero will be reached at a certain point at which $d \xi / d x=0$, implying, with our choice of $x$-coordinate, a maximum of the negative electric field necessary to integrate a positive potential $\Phi_{B}-V$. However, if the IB is thicker, then a zero-field region will appear, at which also $d \xi / d x=0$, as well as the charge density, because the integral of the field is anyway limited to $\Phi_{B}-V$. This behavior can be seen in the band diagram plots in Fig. 3. In the thin IB, the electric field (determined by the slope of the bands) fills the entire IB region, whereas in the thick cell, the electric field is confined to the edges. For these wide IB cells, far from the proximity of the $\mathrm{p}$ and $\mathrm{n}$ regions where electrons or holes are accumulated, the charge neutrality condition is given by $f=N_{D} / N_{t}$ (assuming $N_{D} \leq N_{t}$ ), as deduced from (6).

If the IB is thick, the built-in potential is split into two terms, appearing at the regions elose to the $\mathrm{p}$ and $\mathrm{n}$ regions. If, for instance, $f=N_{D} / N_{t}=1 / 2$, then the built-in potential near the $\mathrm{p}$ region becomes $\Phi_{\mathrm{Bp}}=1.2422-V$ for the 1000-sun wide-IB region IB-CB uncoupled (Example 2), whereas near the $\mathrm{n}$ region, it becomes $\Phi_{\mathrm{Bn}}=\Phi_{B}-1.2422=0.119$ [Fig. 3(c)]. Notice that the IB then behaves as an $n$ region in a $\mathrm{p}-\mathrm{n}-\mathrm{n}^{+}$junction, where the $\mathrm{n}^{+}$region is the $\mathrm{n}$-sandwiching region (often called emitter). When a voltage $V$ is applied, this voltage is sustained by the p-n junction and no external voltage drops at the $n-n^{+}$junction. This has to be taken into account, for instance, for voltage capacilance experiments. On the contrary, if the doping is smaller, below the balance of the IB filling by light and/or thermal escape, we find that the situation is just the opposite. For instance, again in Example 2 


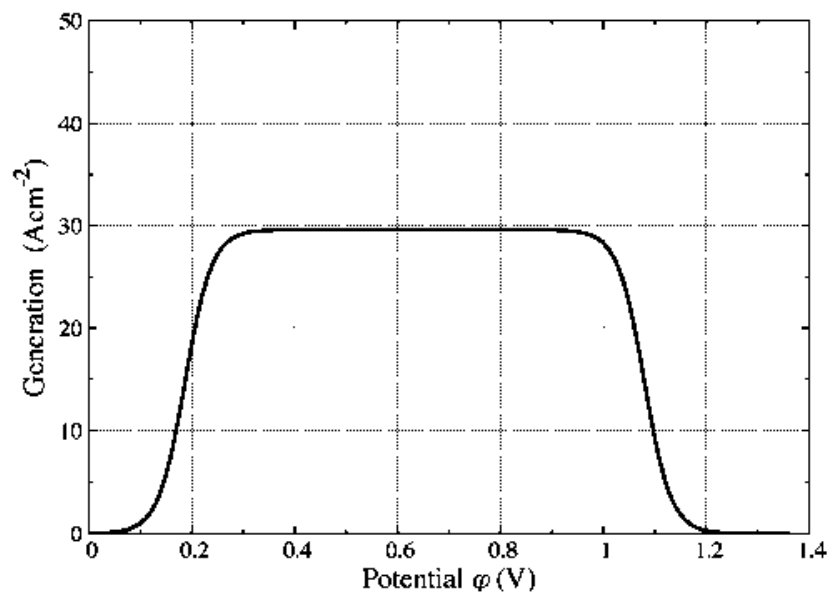

(a)

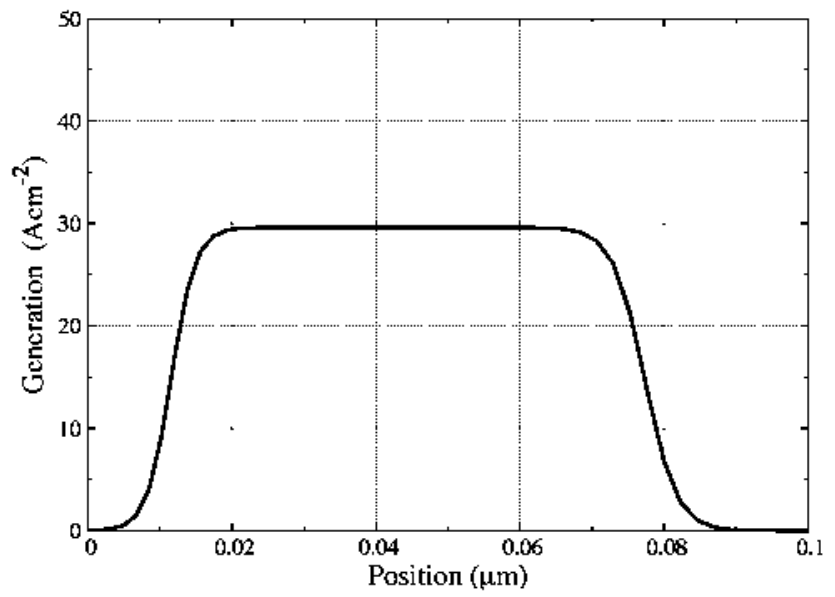

(c)

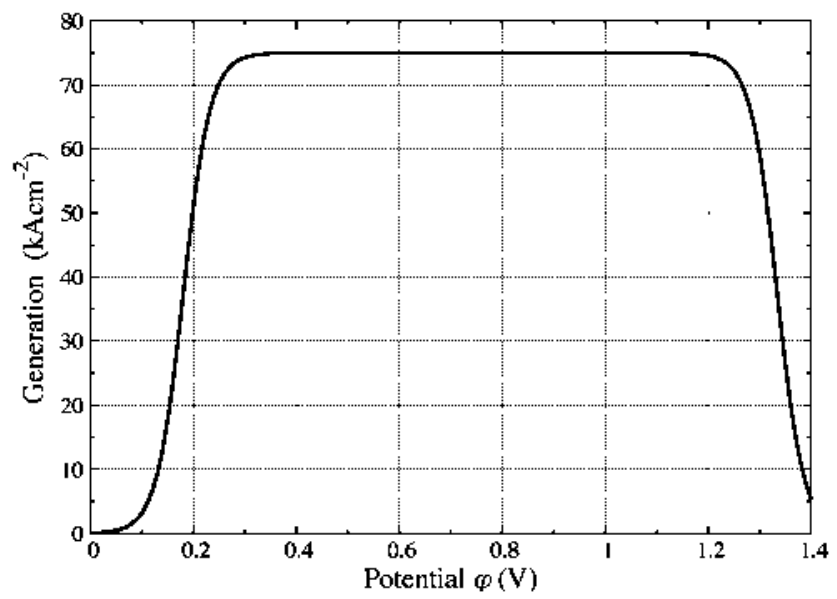

(b)

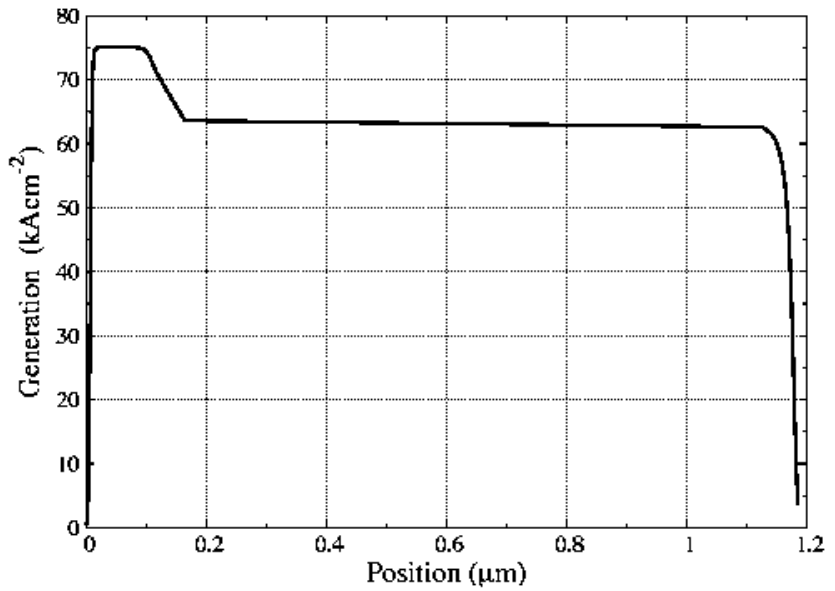

(d)

Fig. 4. Net generation density. (a) and (b) As a function of the electrostatic potential. (c) and (d) As a function of the position. (a) and (c) For the thin IB-CB coupled cell at one sun (Example 1 in Table I). (b) and (d) For the thick IB-CB uncoupled cell under concentrated light (Example 2 in Table I).

(Table I), if $f=N_{D} / N_{t}=0.1$, then $\Phi_{\mathrm{Bp}}=0.1017$ and $\Phi_{\mathrm{Bn}}=$ $1.2596-V$, and the whole structure behaves like a $\mathrm{p}^{+}-\mathrm{p}-\mathrm{n}^{+}$ siructure, where the IB behaves as a p region forming a $\mathrm{p}-\mathrm{n}$ junction at the $\mathrm{n}$ side and a high-low junetion at the $\mathrm{p}$ side.

\section{Generation Profiles}

Based on the filling factor, the generation is given in [12] by

$$
\begin{aligned}
e G=\frac{N_{t} v_{\mathrm{th}}}{f_{d}}\left\{\sigma_{n} \gamma_{\mathrm{l}} N_{C} \exp \left(-\frac{E_{g}-E_{t}}{k T}\right)\right. \\
+\sigma_{p} \gamma_{\mathrm{ln}} N_{V} \exp \left(-\frac{E_{t}}{k T}\right)+\gamma_{\mathrm{lp}} \gamma_{\mathrm{ln}} \\
\quad-\sigma_{n} \sigma_{p} N_{C} N_{V} \exp \left(-\frac{E_{g}}{k T}\right)\left[\exp \left(\frac{V}{k T}-1\right]\right\} .
\end{aligned}
$$

In Fig. 4, we present the generation-potenlial curve at short circuit for the two examples given before. The generation is small in the accumulation regions: on the $\mathrm{p}$ side, because there are no filled states in the IB to produce (thermally or optically) the IB $\rightarrow$ CB transition; on the $\mathrm{n}$ side, because all the IB states are filled and there are no empty states to allow the VB $\rightarrow$ IB transition.
Outside these regions, in the one-sun example (with IB-CB coupled), the first term in the numerator is dominant, being the dominating term in the denominator the one with the same exponential term. Therefore, the generation is given by $e G=e N_{t} v_{\mathrm{ll}_{1}} \gamma_{\mathrm{lp}}=30 \mathrm{~A} \cdot \mathrm{cm}^{-3}$. This represents the fact that the limiting factor for the generation is the VB $\rightarrow$ IB optical transition rate. The second step to the $\mathrm{CB}$ is easily saved by the thermal jump. On the contrary, in the concentration example (Example 2 with IB-CB uncoupled), the dominating term in the numerator is the product of the electron and hole gamma values $\left(\gamma_{l_{n}} \gamma_{1 p}\right)$, not only because the gamma values are about a thousand times larger than at one sun but also because the capture section of electrons is decreased by more than five orders of magnitude. In the denominator, for the same reasons, the terms with the gamma values become dominant. Therefore, $e G=e N_{l} v_{\mathrm{ll}_{1}} \gamma_{\mathrm{lp}} \gamma_{\mathrm{ln}} /\left(\gamma_{\mathrm{ln}}+\gamma_{\mathrm{lp}}\right)=75300 \mathrm{~A} \cdot \mathrm{cm}^{-3}$, expressing the fact that now the generation is limited by the smallest of the optical transition rates between VB $\rightarrow \mathrm{IB}$ and IB $\rightarrow \mathrm{CB}$.

The maximum value in the uncoupled case for $e G$ corresponds to $e G=e N_{t} v_{\mathrm{th}} \gamma_{\mathrm{p}} / 2$ and would be reached when both gamma values are equal. Notice that this maximum is half the value of the maximum value that can be obtained for the coupled IB-CB case. This is so because, in the uncoupled case, the IB is hall-filled, whereas in the coupled IB-CB case, it is emply. 
However, as aforementioned, this wide range of potentials does not correspond to most of the IB space. On the contrary, it has been indicated that most of the IB region, in wide semiconductors, is at the potential that makes the space charge zero, (approximately, the potential for which $f=N_{D} / N_{t}$ ). This explains why, in Fig. 4(d), the region where the generation is maximum is located within the space charge to the left of the neutral zone sinec, in all this neutral zone, the generation is below this maximum because the filing of the IB in this region is not the best, given the values of gamma. In general, the best situation is when the doping level induces a filling factor $f \cong \gamma_{\mathrm{p}} /\left(\gamma_{\mathrm{l}}+\gamma_{\mathrm{n}}\right)=N_{D} / N_{t}$.

One corollary of the preceding arguments is that cells with the IB empty and thermal IB-CB coupling absorb the subbandgap light better than cells with the IB partially filled and uncoupled IB-CB. Indeed, a thicker IB can solve this issue, but at the expense of some additional recombination. Anyway, the splitting of the Fermi level into three quasi-Fermi levels that is clearly visible in open circuil in Fig. 3(d) is not produced in IB-CB coupled IBs [Fig. 3(b)]. Consequently, in IB-CB uncoupled cells, we must rely on a partially filled IB to be able to have empty states to receive the VB $\rightarrow$ IB transitions and full states to supply the IB $\rightarrow \mathrm{CB}$ transitions. The topic has already been discussed in the detailed balance limit [18], [19] analysis of this cell.

\section{Current-Voltage Characteristics}

In the current-voltage characteristics plotted in Fig. 5, which are calculated using the model in [12], both cells have the same parameters, with the following exceptions: 1) The cell in Fig. 5(a) is not doped to prevent the filling of the IB, whereas that in Fig. 5(b) is doped with $N_{D}=0.5 \mathrm{~N}_{t}$. 2) The electron capture cross section $\sigma_{n}$ is $7.83 \times 10^{-13} \mathrm{~cm}^{2}$ in Fig. 5(a) and $7.83 \times 10^{-17} \mathrm{~cm}^{2}$ in Fig. 5(b). 3) The cell thickness in Fig. 5(b) is wider than that in Fig. 5(a) to compensate for the reduction in absorption as a result of the halffilling of the IB. In both cases, light absorption is almost total for below band-gap encrgy photons. The hole capture cross section is low in both cases, but probably achicvable, in both cases being above the value obtained in the detailed balance limit $[20]$.

As it can be seen from the plots, the IB-CB connected cell can produce an increased current, but at the expense of a reduced voltage with respect to an ordinary state-of-the-art GaAs cell [21]. Both factors balance out, and the efficiency is almost unchanged. On the contrary, when IB-CB cells are thermally unconnected and the IB is properly doped, the current can equally be increased by increasing the thickness of the IB region, but the voltage is not reduced as much, and the efliciency can be around or above that of a state-of-the-art double-junction solar cell (32.6\%) [22].

\section{VI, CONCLUSION}

We have examined the role of the IB filling in IB solar cells. The filling factor depends on the potential developed between the $p$ and $n$ regions, which enclose the IB band region, and

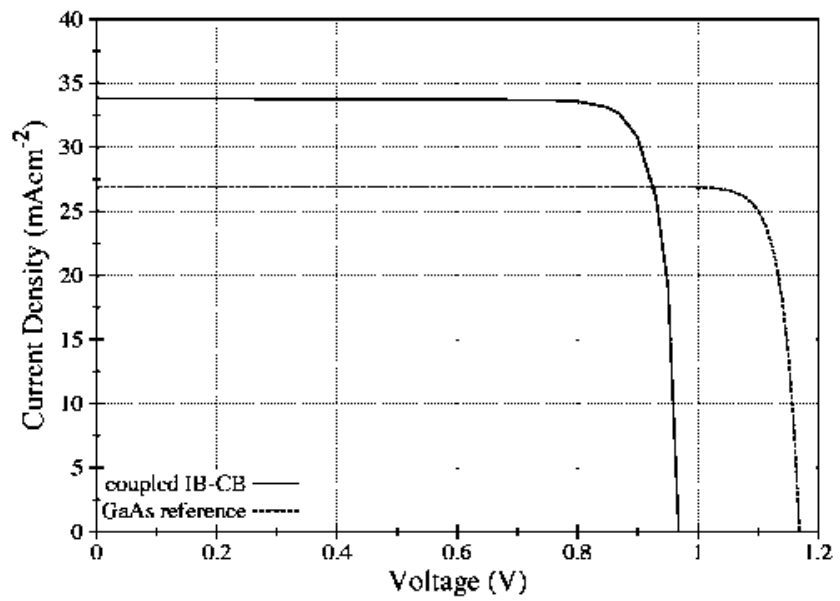

(a)

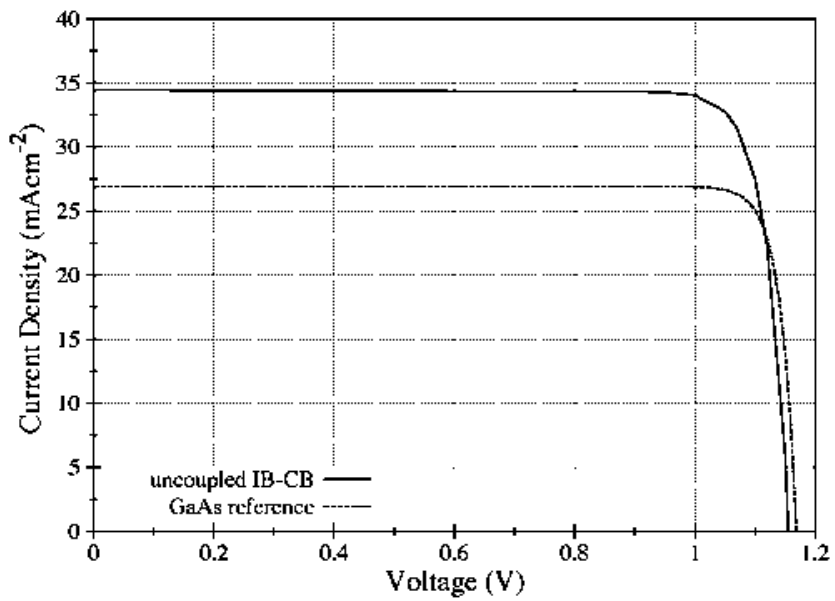

(b)

Fig. 5. Calculated currenl-voltage characteristics at 1000 suns for an IB solar cell (a) with the $\mathrm{lB}$ coupled to the CB (Example 3 in Table l) and (b) with the IB uncoupled to the CB (Example 2 in Table I). In both cases. the characteristics are compared with a state-of-the-art GaAs reference cell. The calculated efficiency values are $28.2 \%$ and $34.2 \%$ for the IBSC for plots (a) and (b), respectively. The efficiency of the GaAs reference cell is $28.1 \%$ (reduced to $26 \%$ in a stale-of-the-art cell [21] due to series resistance).

through the dependence of the potential with the position, it also depends on the position.

For cells where the clectron capture section is very large - we say for this case that the IB is thermally coupled to the $\mathrm{CB}$ - the best situation is to have the IB almost empty to maximize the absorption of photons in the VB $\rightarrow$ IB transition. In this case, it is convenient not to dope the IB with donors. On the other hand, if the capture section is reduced to the extent the $\mathrm{IB}-\mathrm{CB}$ becomes uncoupled and the IB is wide enough, then it must be doped with donors to force a partial filling in most of the IB. The optimal doping is $N_{D}=N_{t} \gamma_{\mathrm{lp}} /\left(\gamma_{\mathrm{lp}}+\gamma \mathrm{ln}\right)$.

It is confirmed once again that, while with the appropriate IB thickness the IB-CB coupled cell may increase its current, it does it at the cost of reducing the voltage. The result is that, once all technological intricacies are solved for the coupled QD InAs/GaAs solar cells, we can only expect efficiency values similar to good $\mathrm{GaAs}$ solar cells. On the contrary, if the $\mathrm{IB}-\mathrm{CB}$ in a QD InAs/GaAs cell is uncoupled, then the efficiency under concentrated light of the same structure may be in the range of the best dual-junction tandem cells also under concentrated 
sunlight. The total potential barrier is set by the doping of the enclosing semiconductors. When the IB is wide, the IB region is made up of a neutral zone surrounded by two space-charged zones. When doping above the optimal value, most of the potential barrier is developed in the p-IB junction, and this partial barrier is the one variable with the applied voltage. In this case, the IB-n potential barrier is constant. This behavior-for $C-V$ measurements for instance-is like that of a $p^{+} n-n^{+}$structure. On the contrary, below the optimal doping, the situation is reversed: the highest voltage potential barrier is at the IB-n interface, and the $\mathrm{p}^{+} \mathrm{IBn}^{+}$structure behaves like a $\mathrm{p}^{+}-\mathrm{p}-11^{+}$ structure.

\section{REFERENCES}

[1] A. Luque and A. Martí, "Increasing the efficiency of ideal solar cells by photon induced transitions at intermediate levels," Phys. Rev. Lett. vol. 78, no. 26, pp. 5014-5017. Jun. 1997.

[2] A. Luque and A. Martí. "A metallic intermediate band high efficiency solar cell." Prog. Photovolt. Res. Appl., vol. 9. no. 2, pp. 73-86. Apr. 2001.

[3] M. Wolf. "Limitations and possibilities for improvements of photovoltaic solar energy converters. Part I. considerations for Earth's surface operation," Proc. IRE. vol. 48, no. 7. pp. 1247-1263, Jul. 1960.

[4] M. J. Keevers and M. A. Green, "Efficiency improvements of silicon solar cells by the impurity photovoltaic effect," $J$. Appl. Phys., vol. 75. no. 8 . pp. 4022-4031, Apr 1994.

[5] A. S. Brown and M. A. Green, "Impurity photovoltaic effect: Fundamental energy conversion efficiency linits." J. Appl. Phys., vol. 92, no. 3. pp. 1329-1336, Aug. 2002.

[6] A. S. Brown and M. A. Green, "Impurity photovoltaic effect with defect relaxation: Inplications for low band gap semiconductors such as silicon," J. Appl. Phys. vol. 96, no. 5, pp. 2603-2609, Sep. 2004.

[7] A. Lugue, A. Martí, E. Antolín, and C. Tablero, "Intermediate bands versus levels in non-radiative recombination." $P$ hys. $B$, vol. 382 , no. $1 / 2$. pp. 320-327, Jun. 2006.

[8] D. V. Lang and C. H. Henry, "Nonradiative recombination at deep levels in GaAs and GaP by lattice-relaxation multiphonon enission." Phys. Rev. Lett., vol. 35, no. 22, pp. 1525-1528, Dec. 1975.

[9] E. Cánovas, A. Martí, N. López, E. Antolín, P. G. Linares, C. D. Farmer, C. R. Stanley, and A. Luque, "Application of photoreflectance technique to the characterization of quantum dot intermediate band materials for solar cells." Thitt Solid Films, vol. 516, no. 20. pp. 6943-6947, Aug. 2008

[10] V. Popescu. G. Bester. M. C. Hanna, A. G. Norman, and A. Zunger, "'Theoretical and experimental examination of the intermediate-band concept for strain-balanced (In,Ga)As/Ga(As,P) quantum dot solat cells," Phys. Rev. B. Condens. Matter. vol. 78, no. 20. p. $205321-17$. Nov. 2008.

[11] S. Tomic. T. S. Jones. and N. M. Harrison. "Absorption characteristics of a quantum dor array induced intermediate band: Implications for solar cell design," Appl. Phys. Lett., vol. 93, no. 26, p. 263105. Dec. 2008.

[12] A. Luque. A. Martí, N. López. E. Antolín. E. Cánovas. C. Stanley. C. Farmet, and P. Diaz, "Operation of the intermediate band solar cell under nonideal space charge region conditions and half filling of the intermediate band," J. Appl. Phys., vol. 99, no. 9. p. 094503. May 2006.
[13] R. Strandberg and T. W. Reenaas, "Photofilling of intermediate bands," $J$. Appl. Phys. vol. 105, no. 12, p. 124512. Jun. 2009.

[14] A. S. Lin, W. Wang, and J. D. Phillips. "Model for intermediate band solar cells incorporating cartier transport and recombination." J. Appl. Phys. vol. 105. no. 6, p. 064512, Mar. 2009.

[15] W. Shockley and W. T. Read, "Statistics of the recombination of holes and electrons." Phys. Rev. vol. 87. no. 5, pp. 835-842. Sep. 1952.

[16] R. N. Hall, "Electron-hole recombination in germanium." Phys. Rev. vol. 87, no. 2, p. 387, Jul. 1952.

[17] Y. K. Hsieh and H. C. Card, "Limitation to Shockley-Read-Hall model due to direct photoionization of the defect states." J. Appl. Phys., vol. 65. no. 6, pp. 2409-2415, Mar. 1989 .

[18] A. Luque, A. Martí, and L. Cuadra, "Thermodynamic consistency of subbandgap absorbing solar cell proposals," IEEE Trans. Electron Devices. vol. 48, no. 9. pp. 2118-2124, Sep. 2001

[19] A. Luine, A. Martí, and L. Cuadra, "Thermodynamics of solar energy conversion in novel structures." Phys. E, vol. 14, no. 1/2, pp. 107-114. Apr. 2002.

[20] W. Vanroosbroeck and W. Shockley, "Photon-radiative recombination of electrons and holes in germanium." Phys. Re., vol. 94. no. 6. pp. 15581560. Jun. 1954.

[21] C. Alyora, E. Ortiz, I. Rey-Stolle, V. Díaz, R. Pea, V. M. Andreev, V. P. Khvostikov, and V. D. Runyantsev, "A GaAs solar cell with an efticiency of $26.2 \%$ at 1000 suns and $25.0 \%$ at 2000 suns." IEEE Trans. Electron Devices, vol. 48, no. 5. pp. 840-844, May 2001.

[22] I. García. I. Rey-Stolle, B. Galiana. and C. Algora "A 32.6\% efticient lattice-matched dual-junction solar cell working at 1000 suns." Appl. Phys. Letl., vol. 94, no. 5, p. 053509, Feb. 2009.

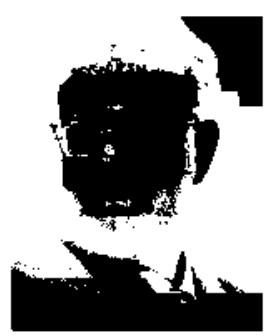

Antonio Luqne (S'62-M'77-SM'77) recejved the Doctor Ingeniero de Telecomunicación from the Universidad Politécnica de Madrid, Madrid. Spain. in 1969.

He has been a Full Professor of electronic technology with the Universidad Politécnica de Madrid since 1970 and is a fonnder of its Institute of Solar Energy in 1979. His research activity is devoted to photovoltaics. In 1981. he created the solar cells company Isofotón. with a turnover of 297 million euros in 2007

Prof. Luque has been the recipient of several important distinctions for photovoltaic reseatch.

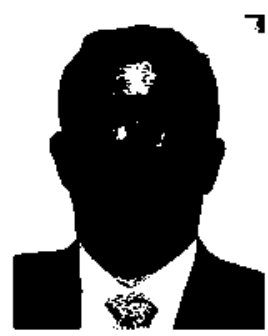

Antonio Martí recejyed the Ph.D. degtee from the Universidad Politécnica de Madrid, Madrid. Spain. in 1992 .

He has been a Full Professor of electronic technology with the Universidad Politécnica de Madrid since 2007. Since 1997. he has been collaborating with Prof. L. in the development of the intermediateband solar cell 